\title{
Risk Assessment of Information System
}

\author{
Mounica Durba1)
}

\begin{abstract}
The main purpose of the paper is to scale back the risks in data systems. associate system is any organized system for the gathering, organization, storage and communication of knowledge. System is an instructional study of systems with a selected regard to data and also the complementary networks of hardware and software system that individuals and organizations use to gather, filter, process, produce and conjointly distribute information. Information systems have long been in danger from malicious actions, accidental user error, natural disasters and alternative unforeseen adverse events.
\end{abstract}

Keywords : risk, assessment, information system, defence level, risk value

\section{Introduction}

Enterprise info systems are the big complicated software package systems and tools underlying several of the university's core body and academic support processes. Examples embrace the systems related to finance, analysis support, human resources, student administration, course management, and several other others. On a daily basis these systems method thousands of business transactions within which information are entered, manipulated, and keep for each operational and informational functions. The ensuing info resources represent a valuable institutional plus that's used for analysis and deciding.

Enterprise info systems have each operational (transaction-processing) and informational (data storage, retrieval, and reporting) functions. The operational goals tend to be targeted on potency. once done well, enterprise info systems build it easier for folks to finish their add a timely and correct manner. They additionally modify the university to standardize and contour its operations. against this, poor quality enterprise systems will truly increase workloads, cause frustration among the those that use them, and cause pricey operational disruptions. The informational goals of enterprise systems concentrate on effectiveness. Institutional information has to be pronto offered to decision-makers during a type that marries information with

Received(May 11, 2015), Review Result(1st: May 28, 2015, 2nd: June 25, 2015), Accepted(September 10, 2015)

${ }^{1}$ (Corresponding Author) Department of Computer Science and Engineering, KL University email: mounica.dr96@gmail.com 
context. The quality of the data is additional vital than the amount of information. Like several universities, Cornell has found it difficult to deploy and support these systems in ways in which optimally balance operational potency with informational effectiveness. it's troublesome for workers to keep up the depth and breadth of technical skills required by our heterogeneous multi-platform atmosphere. The prices related to implementing and supporting these systems is substantial. It will take an extended time to put in or upgrade them. they're complicated and may be troublesome for folks to use, particularly for people who use bound systems sometimes however below tight deadlines, as is that the case for several researchers.

Enterprise systems represent the university's single largest space of investment in software package applications. Several if not most of our employees, faculty, and students act with one or additional of those systems on a daily basis. The mixture of the substantial price and widespread use of enterprise systems makes it a matter of urgency to enhance the cost-benefit (or worth proposition) of this cluster of systems. this may be done by reducing the price or up the advantages, or both. additionally, it's vital that these systems replicate economical and effective business practices, which they not simply modify existing, typically cumbersome, processes. To align with a analytic thinking this objective is split into 2 distinct sections initiatives to decrease price and initiatives to extend business worth.

Informed, data-driven deciding depends on correct, timely, and welldefined info resources. whereas it's going to not be possible, or maybe fascinating, to possess one technology resolution to support all of Cornell's "institutional intelligence" wants, it's very important that the establishment perceive the characteristics of its information resources through applicable governance, policies, and a coherent design.

Many points of integration and resource dependencies exist among our enterprise info systems. A deficiency in one system will have an effect on another system that depends on shared information or workflow. Managing multiple software package platforms, versions, upgrades, associated implementation comes needs that we have a tendency to read these systems not solely on an individual basis however additionally as an integrated portfolio that acknowledges the interdependencies.

Especially, in recent years, systems is turning into additional liable to these threats because of the increasing interconnectivity of laptop networks and additional mutualist and accessible to an oversized variety of people. We have a tendency to decision the absence or weakness of a safeguard in some plus or resource the vulnerability of the system. The absence or defect makes a threat or attack doubtless additional harmful or pricey and additional possible to occur. So as to avoid the protection event happening, several measures is enforced. Wherein, 
risk assessments, whether or not they pertain to data security or alternative varieties of risks, area unit means that of providing call manufacturers with the knowledge required to grasp factors which will negatively influence operations and outcomes, and build wise to judgments regarding the extent of actions required to scale back risk. Risk in any context is that the total of threats (those events that cause harm), vulnerabilities (the openness to the threats) and plus price (the value of the plus in danger). a rise in any of those factors will increase the danger.

The aim of risk assessment is to grasp the current and future system risks, access the protection threats and also the degree of influence in all probability engendered from these risks, and supply the premise for security strategy identification, institution and safe operation of the knowledge system. There area unit 2 common approaches employed in risk assessment: a quantitative approach and a qualitative approach. Many countries and organizations have established the danger assessment audit standards and also the principle of risk calculation is given because the follows:

$$
\text { Risk }=\mathrm{R}(\mathrm{A}, \mathrm{T}, \mathrm{V})=\mathrm{R}(\mathrm{L}(\mathrm{T}, \mathrm{V}), \mathrm{F}(\mathrm{I} \mathrm{a}, \mathrm{V} \text { a }))
$$

Quantitative and qualitative strategies are 2 elementary teams of strategies ar applied for analysis of risk on that assets are exposed in a corporation. The foremost necessary blessings and drawbacks of it risk assessment strategies are given[1].

Quantitative wherever estimation of risk price is connected with application of numerical measures- price of resources is outlined in quantity the frequency of threat incidence within the variety of cases and susceptibleness by the worth of chance of its loss, those strategies gift leads to the form of indicators. The samples of quantitative strategies Annual loss expected, Courtney's and Fisher's strategies, ISRAM model etc[2-5].

Qualitative that don't care for numerical knowledge presenting leads to the shape of descriptions, recommendations wherever assessment is connected with. Qualitative description of assets price, determination of qualitative scales for the frequency of threat incidence and susceptability for a given threat or description of therefore known as threat situations by prediction of the most risk factors. The samples of qualitative strategies FMEA/FMECA, The Microsoft company security risk management Framework, CRAMM[6-8]. relying upon the seriousness of given threat there are often applied totally different risk measures from terribly straightforward assessments, determining the chance as high, medium and low to terribly precise indicators given as chance of given event occurences.

On this data security risk assessment strategies are often classified 2 categories: One is that 
the ancient risk assessment strategies, like FMECA, HAZOP, Markov so on; the opposite is that the trendy strategies of risk assessment, like CORAS, CRAMM, COBIT so on. Zhang et al. provides a model that thought-about the link between totally different vulnerability, and will discover the hidden risks. however of these strategies take into account the vulnerability and also the threat individually for distinguish equipments rather than regarding the result among the equipments. High interconnection between threat objects is one in every of characteristics of data system. There are some security dependency relationships between threat objects in line with the safety methods. Threat might complete Associate in Nursing attack mistreatment isolated vulnerabilities. Then there's correlation between vulnerabilities. At present, the correlation between vulnerabilities is employed by most of the threat that are for the knowledge system. Produce conditions for mistreatment next vulnerability by mistreatment the vulnerability. During this paper we tend to take into account the result among the equipments within the system and gift a risk assessment technique, by that we will calculate the vulnerability and threat with efficiency and improve the accuracy of the results of risk assessment.

\section{Threat and Vulnerability}

In pc security a threat may be a attainable danger which may exploit a vulnerability to breach security and therefore cause attainable hurt. A threat are often either "intentional" (i.e., intelligent; e.g., a personal cracker or a criminal organization) or "accidental" (e.g., the chance of a pc run-down, or the chance of a natural disaster like associate degree earthquake, a fire, or a tornado) or otherwise a circumstance, capability, action, or event. People are often inquisitive about finding out all attainable threats that can affect associate degree plus, have an effect on a software package square measure brought by a threat agent.

By the standard methodology, we will denote a data system as a graph $G(D, E)$, wherever $D$ is vertex set representing the set of equipment's and also the $\mathrm{E}$ is edge set indicating the relationship of parts of $D$. Given associate degree data system $G(D, E)$, for $d, d j \in D$, They are connected by $\mathrm{e} \in \mathrm{E}$, we all know $d i$ and $d j$ should affected directly one another. If there exists another instrumentation that connect with $d$ by $\mathrm{e}$, by constant reason, $d j$ is vulnerable too. Next, we'll construct threat and vulnerability matrix exhibiting the impact relationship. 
The qualitative risk assessment method employed in this risk assessment methodology uses the C-I-A Triad model to assist confirm risk to info resources. C-IA represents Confidentiality, Integrity, and availableness, which are the core principles of data security.

\section{Confidentiality:}

When protective info, we would like to be able to limit access to people who area unit allowed to envision it; everybody else ought to be disallowed from learning something concerning its contents. This is often the essence of confidentiality. For instance, federal law needs that universities limit access to personal student info. The university should take care that solely people who area unit approved have access to look at the grade records.

\section{Integrity:}

Integrity is that the assurance that the data being accessed has not been altered and really represents what's meant. Even as an individual with integrity means that what he or she says and might be trustworthy to systematically represent the reality, data integrity means that data really represents its meant that means. Data will lose its integrity through malicious intent, like once somebody who isn't licensed makes a modification to purposely misrepresent one thing. An example of this could be once a hacker is employed to travel into the university's system and alter a grade.

Integrity may also be lost accidentally, like once a computer power surge corrupts a file or somebody licensed to form a modification accidentally deletes a file or enters half-truths.

\section{Availability:}

Information handiness is that the third a part of the Central Intelligence Agency triad. Handiness means info are often accessed associated changed by anyone approved to try to therefore in an acceptable time frame. Counting on the kind of knowledge, acceptable time frame will mean various things. For instance, a bargainer desires info to be offered at once, whereas a sales person is also happy to urge sales numbers for the day in a very report successive morning. Firms like Amazon.com would require their servers to be offered mean solar day every day, seven days every week. Different firms might not suffer if their internet servers are down for many minutes once in a very whereas. 
1. Every resource is classed in line with level of confidentiality (Confidential, Sensitive, Public) and criticality (Mission essential or not).

2. Resources are sorted along for assessment, and every assessment is assigned associate overall rating for Confidentiality, Integrity, and handiness.

3. Assessors answer queries as a part of the assessment method. These queries verify whether or not needed operational, technical, or body security controls are gift. every question is assigned a base rating for Confidentiality, Integrity, and handiness. so as to see inherent risk.

4. The inherent risk for every question is calculated victimization the bottom C-I-A ratings and also the unit-assigned overall C-I-A ratings. This enables the inherent risk for every question to be somewhat custom-made to a unit atmosphere and business perform. Inherent risk is that the level of risk gift before application of a compensating security management.

Vulnerability may be a weakness that permits an (attacker) to cut back a system's (information assurance). Vulnerability is that the intersection of 3 elements: a system susceptibleness or flaw, assailant access to the flaw, and assailant capability to use the flaw. To use a vulnerability, associate assailant should have a minimum of one applicable tool or technique which will hook up with a system weakness. During this frame, vulnerability is additionally referred to as the (attack surface. Vulnerability management is that the diurnal apply of distinctive, classifying, re-mediating, and mitigating vulnerabilities. This apply usually refers to computer code vulnerabilities in computing systems. A security risk could also be classified as a vulnerability. The employment of vulnerability with a similar that means of risk will result in confusion. The danger is tied to the potential of a major loss. Then there area unit vulnerabilities while not risk: as an example once the affected (asset) has no worth. A vulnerability with one or a lot of better-known instances of operating associated absolutely enforced attacks is assessed as an exploitable vulnerability, a vulnerability that an (exploit) exists.

Definition: Given associate system $G(D, E)$ with $|D|=m$, we tend to decision the matrix $T$ the ballroom dance vulnerability result matrix

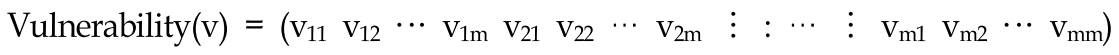

where $V_{i j}$ denotes the likelihood associate attack intrudes into instrumentation $d_{j}$ beneath 
the condition the attack has conquered the instrumentation $d_{i}$ and $d_{j}$ includes a special vulnerability. For the on top of matrixes, $\mathrm{T}-\mathrm{I}$ denotes the ballroom dance threat result matrix between completely different equipment's, wherever I may be a identity matrix that order is same with T. Similarly, V - I is one-step vulnerability matrix between completely different equipment's.

Consistent with the analysis, we all know that any try of kit $\mathrm{d}_{i}, \mathrm{~d}_{j}$ will be affected one another if there exists a path $\mathrm{d}_{j} \mathrm{~d}_{i+1}, \ldots . \mathrm{d}_{i+1}, \mathrm{~d}_{j}$. For the trail, if associate instrumentation $\mathrm{d} \mathrm{m}$ is passed doubly, then deleting the cycle of the trail, the reside path is additionally a path from $\mathrm{d}_{i}$ to $\mathrm{d}_{j}$ therefore we will notice associate path while not cycles between $\mathrm{d}_{i}$ and $\mathrm{d}_{j}$ applying the on top of methodology recursively. That the result of $\mathrm{d}_{j}$ brought by di ought to be calculated. In order to calculate the result between equipment's, we tend to provide the subsequent operation rule.

\section{Risk Assessment}

According to the chance formula one, once determinative the worth of kit, mix the higher than vulnerability and threat, we will acquire the chance level of a data system. We find the results of our projected methodology is coincide with the results of the classical risk assessment methodology. On the opposite hand, our methodology amplify the chance price, particularly for the threat with high risk price within the ancient methodology that is helpful to conduct the directors to deploy the economical defence strategy.

$$
\begin{aligned}
& \text { Risk }=\mathrm{R}(\mathrm{A}, \mathrm{T}, \mathrm{V})=\sum \text { Pth th }(\text { Ath }) \mathrm{T} \\
& \text { Pth }=\text { Tc.Vc }=\left\{\begin{array}{llllll} 
& c . v 1 & c, 2 & c . v 2 & c, \cdots \cdots, t m & c . v m c
\end{array}\right\}
\end{aligned}
$$

\section{Implementation Algorithm}

Step1: Determine the assessed information system,

Author considered a typical enterprise information system that consists of Information center. Admin server, Applications, User Group 1, User Group 2.

In the above diagram

e1 - information system

e2 - admin server 
e3 - Applications
e4 - User Group 1
e5 - User Group 2.

i.e for the information $G(D, E)$

Where $\mathrm{D}=\{\mathrm{e} 1, \mathrm{e} 2, \mathrm{e} 3, \mathrm{e} 4, \mathrm{e} 5\}$

The information system is divided into levels for reducing the complexity. The levels are divided based on their roles, asset values, possible threats and vulnerabilities. Level 1: Information Center Level 2: Admin Server Level 3: Applications Level 4: User Group 1 Level 5: User Group 2

Step2: Identify the assets,

The information center is denoted as e1, the administrator server as e2, the Applications as e3, the User Group 1 as e4, the User Group 2 as e5 respectively, i.e. for the information G(D, E), $D=\{$ e 1 , e 2, e 3 , e 4 , e 5$\}$

By using the tool and queries, we find the system has the following threats:

th1: external Dos attack,

th2: malicious code,

th3: disorder of management,

th4: information sniffers.

In our experiment, we only consider the first four threats, so $T=\{$ th1, th 2 , th 3 , th 4$\}$.

Calculation of Asset Valuation Matrix is based on the following methodology

1. Confidentiality, Integrity and Availability of information will have minimum valuation.

2. The value of levels for Confidentiality, Integrity and Availability are taken as

Follows: Low 1, Medium 2, High 3

3. Asset value is determined by sum of all (attribute * its level).

4. Example

Confidentiality Integrity Availability Asset Value 
High High High $3+3+3=9$

Medium Medium Medium $2+2+2=6$

Low Low Low $1+1+1=3$

High Medium Low $3+2+1=6$

C Low Medium High

I L M H L M H L H

A Low 345456567

Medium 456567678

High 567678789

Severity and Threat Vulnerability Matrix is based on the following methodology.

The value of levels for severity of threat and vulnerability are taken as follows: Low 1, Medium 2, High 3

The severity value matrix will be mathematical Asset Value * Severity of Threat Value * Severity of Vulnerability Value.

Example

Asset Value

Severity of Threat

33696121891827

4481281624122436

$\begin{array}{llllllllll}5 & 5 & 10 & 15 & 10 & 20 & 30 & 15 & 30 & 45\end{array}$

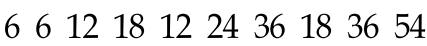

$\begin{array}{lllllllll}7 & 14 & 21 & 14 & 28 & 42 & 21 & 42 & 63\end{array}$

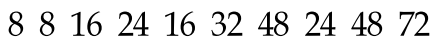

$991827 \quad 183654275481$

Probability

Value Explanation Example

1 Never happened Not happened in last 3 years

2 Rare Once in Year

3 Periodic Once in a Quarter

4 Regular Once in a fortnight

5 Frequent Once in a week

Asset Value

Severity of Threat 
Severity of Vulnerability

Probability

5 High $=3$ Low $=1$ Periodic $=3$

7 Medium $=2$ High $=3$ Regular $=4$

8 Medium $=2$ High $=3$ Regular $=4$

9 High $=3$ High $=3$ Frequent $=5$

Step3: Detect the logical relation of assets, Identify the vulnerability of the assets, and evaluate initial vulnerability vector V I, Where $\mathrm{D}=\{\mathrm{e} 1, \mathrm{e} 2, \mathrm{e} 3, \mathrm{e} 4, \mathrm{e} 5\}$.

The information system is divided into levels for reducing the complexity. The levels are divided based on their roles, asset values, possible threats and vulnerabilities. Level 1: Information Center Level 2: Admin Server Level 3: Applications Level 4: User Group 1 Level 5: User Group 2

\section{Conclusion:}

We find the values of their vulnerability increase $130 \%$ and $138 \%$ of the equipment 1 and equipment 3 respectively. By the infrastructure of the information system, we know the equipment 2 is connected with equipment 1 and equipment 3 . By improving the defence level of equipment 2, the result of assessment shows the risk decrease drastically.

Applying risk calculation (6), we obtain the risk value under threat th 1 is 72.3482 and the risk value is 4.5 without considering the correlation of equipment's. Applying the same method, we obtain all risk value of other threats in Fig. 3. From the Fig. 3, we find the result of our proposed method is coincide with the result of the classical risk assessment method. On the other hand, our method magnify the risk value, especially for the threat with high risk value.

\section{References}

[1] S. K. Pandey, K. Mustafa, A Comparative Study of Risk Assessment Methodologies for Information Systems, Bulletin of Electrical Engineering and Informatics, (2012), Vol.1, No.2, pp.111-122.

[2] Mohamed Ghazouani, Sophio Faris, Information Security Risk Assessment - A Practical Approach with a Mathematical Formulation of Risk, International Journal of Computer Applications (0975-8887), (2014), Vol.103, No.8.

[3] Daniyal M. Alghazzawi, Syed Hamid Hassan, Mohammed Salim Trigui, Information Systems Threats and 
Vulnerabilities, International Journal of Computer Applications (0975 - 8887), (2014), Vol.89, No.3.

[4] J. Zhang Y. Zheng, Research on Network Node Correlation in Network Risk Assessment", China Journal of Computers, (2007), pp.234 240.

[5] Y. P. O. Yaang, H. M. Shieh, and G. H. Tzeng, A VIKOR technique based on DEMATEL and ANP for information security risk control assessment, Information Sciences, (2013), Vol.232, pp.482-500.

[6] K. J. Farn, S. K. Lin, and C. C. Lo, A study on e-Taiwan information system security classification and implementation, Computer Standards \& Interfaces, (2008), Vol.30, No.1, pp.1-7.

[7] S. G. René, Information security management best practice based on ISO/IEC 17799, Information Management Journal, (2005), Vol.39, No.4, pp.60-66.

[8] D. Ergu, G. Kou, Y. Shi, and Y. Shi, Analytic network process in risk assessment and decision analysis, Computers \& Operations Research, (2014), Vol.42, pp.58-74. 